\title{
An application of microprocessor technology to automated data collection
}

\author{
LAWRENCE J. FITZPATRICK \\ Institute of Child Development, University of Minnesota, Minneapolis, Minnesota 55455
}

\begin{abstract}
This paper describes the application of a low-power 8-bit microprocessor and its supporting hardware to the collection and transmission of binary data in the ELOG data collection system. It includes a discussion of the way ELOG functions and the hardware used to support that function, as well as a description of the computer algorithms used to organize data collection.
\end{abstract}

There are a number of possible ways to enter time-based event records into a computer to be stored for subsequent analysis, and for each way there are a number of hardware configurations with which to do the job (Fitzpatrick, 1977). The range of acceptable hardware for data entry is limited, however, if collection of data at remote sites and portability of equipment are important study objectives. The advent of microprocessors has permitted greatly improved portability in systems for remote data entry by making it possible to reduce the size and power of input systems. Microprocessors are small and use little power, but more importantly, they have the ability to do all the intelligent things at the remote site necessary to allow elimination or replacement of power-consuming system components. The present article describes the hardware and algorithms used in the ELOG data acquisition system, a microprocessor application developed by the author for automated data collection.

\section{GENERAL DESCRIPTION}

The ELOG data acquisition system is designed to record data at remote sites and then to transmit it to a central computer for processing via a 300-baud (30 characters/sec) timesharing port. A brief overall description of the architecture and use of the system was given by Fitzpatrick (1977).

The system includes a rechargeable battery pack that uses six D-size nickel-cadmium batteries and can provide up to $8 \mathrm{~h}$ of continuous battery operation, the ELOG controller, which gets its power from the battery pack and includes an 8-bit microprocessor, 512 words of read-only memory (ROM), 256 words of random-access memory (RAM), and the required input/output interfaces: a simple 6-V monaural cassette recorder (GE Model 3.5001 A, which retails for under $\$ 25$, for instance) powered by the battery pack, and up to 20 input key switches organized as four individual five-key "keyboards." The system uses a single microprocessor (National Semiconductor ISP 8A/600, SC/MP II) with separate algorithms for data recording and data transmission. A complete description of this micro- processor, including schematics and program instruction set, is available from the manufacturer (National Semiconductor Corporation, Note 1). The battery pack, ELOG controller, and cassette recorder are used for data recording and transmission, while the keyboards are used only in data recording. The battery pack is provided with a power switch, and the controller has four switches with two positions each for user control: a power switch (on-off), a timer switch (on-off), a mode switch (transmit-record), and a momentary reset switch (reset-null). The controller uses CMOS components wherever possible to minimize power consumption. National Semiconductor component numbers are used to specify integrated circuits; in most cases a number of manufacturers produce equivalent parts. Detailed descriptions of hardware, function, and algorithms are presented below.

\section{ELOG HARDWARE AND FUNCTION}

\section{Data Recording}

The user initializes the system by setting the timer switch to "off" and the mode switch to "record." He begins recording by turning on the battery pack and controller power and then holds the reset switch depressed to provide power to the cassette recorder while he positions the cassette at its starting location. The keyboards are connected and the user begins a recording session by depressing the timer switch. This switch activates a precision R-C timer (LM 2905) that "ticks" every $.1 \mathrm{sec}$ and, with each tick, requests an interrupt that directs the microprocessor to scan the keyboard. The system uses parallel-mode data input. This means that each key operates independently of every other key and any or all keys can be depressed at a given time. All 20 keys are read each $.1 \mathrm{sec}$ while the timer switch remains on. In addition, a synchronizer signal that uses a bandpass filter (LM 567) set at $3,000 \mathrm{~Hz}$ to simulate a switch closure is monitored as Key 21 .

The keyboards are connected to the inputs of three parallel-in, serial-out, 8-bit shift registers (74C 165) with pull-up resistors on each input to minimize noise. 
These shift registers are connected serially, and the microprocessor reads the keyboard information through its "serial-in" port. To minimize data storage requirements, the microprocessor checks for changes in key depressions from the last keyboard configuration read. When a key change occurs, the last keyboard configuration and its duration are stored in the 256 by 8 RAM ( 2 each: 256 by 4 CMOS RAM, 74C 921). When the data buffer in the RAM contains 176 words, the cassette recorder is activated and the data in the buffer is placed on cassette tape. The microprocessor controls the recorder through one of its "flag" ports by means of a Darlington switch (General Electric D34C6). A full data buffer can be placed on tape in approximately $5 \mathrm{sec}$, and keyboard data occurring while the buffer is being transferred is simply added at the end of the buffer. Sixteen key changes in the 2 sec immediately following recorder activation are necessary to cause buffer overflow, so there is little chance of data loss with this design.

The format in which data is sent to tape is described by Fitzpatrick (Note 2), and the hardware is quite simple. The microprocessor sends 4 bits at a time via its Data Pins 0-3. The 4 bits are first loaded into a quad-D flip flop (74C 175) using a microprocessor store instruction, and the flip-flop output sets the resistance values on a quad electronic switch (CD $4066 \mathrm{C}$ ). Both halves of a dual oscillator (NE 556) are then used to create the audio pulse. The combined resistance across the quad switch determines the "off" time of one oscillator set in a monostable configuration, and this off time then controls the number of pulses generated by the other oscillator set in an astable configuration. These pulses are sent to the recorder. The astable frequency is chosen so that the square-wave astable pulses set up minimal harmonic interference in the simple cassette recorder.

The cassette recorder is automatically activated and data is transferred each time the data buffer fills. When the user has completed a recording session, he turns off the timer, and this signals the microprocessor to transfer all remaining data to tape. A data record begins with two coded "start" words. Each data buffer ends with three "stop" words, and a complete record is concluded with a special code in these three words.

\section{Data Transmission}

The user initializes the system in transmit mode by setting the timer switch to "off" and the mode switch to "transmit." He then connects the ELOG controller to the current loop interface of the modem using the RS 232 connector that usually appears above the timesharing terminal connections. The controller is optically coupled to the modem in both directions. The user initiates transmission by calling the central data receiving computer from a 200-baud timesharing terminal via modem and telephone. He then initiates execution of the data receiving program in the central computer. Next, he turns on the ELOG controller, depresses and holds the reset switch, and positions the cassette tape at its starting location.

From this point, data transmission proceeds automatically. The cassette recorder continues to run after the reset switch is released, and data is transmitted from the recorder back to the microprocessor. The data is read through a simple high-pass capacitor-transistor filter (.01-microfarad mylar capacitor and Fairchild 2N3642) that screens out low-frequency cassette noise. The filter output is fed through a CMOS inverter $(74 \mathrm{CO4})$ that squares and inverts the signal, and it is finally delivered to the interrupt pin of the microprocessor. The microprocessor studies the timing and number of interrupt pulses to recover the data previously placed on cassette. The data is once again stored in the data buffer, and when the cassette has been read through the end of a buffer segment, the controller stops the cassette recorder. The microprocessor then validates the buffer information, and if the data is valid, it is transmitted across the modem to the central computer. It is sent from the serial-out pin of the microprocessor via an optoisolator (General Electric H11B1) to the $20 \cdot \mathrm{mA}$ receive loop of the modem. The data is transferred in binary format as the low 4 bits of an 8-bit word, so that transmission can be effected across most timesharing ports independently of their input/output formats. Translation from binary is simply written into the data receiving program used by the host computer.

After receiving a buffer of data, the host computer acknowledges receipt and verifies that there were no mistakes in transmission. These signals are transferred back to the controller across the 20-mA transmit loop of the modem and through a second optoisolator (General Electric H11A10) to the serial-in pin of the microprocessor. Data continues to be transmitted buffer by buffer until a complete data record has been transferred and a stop code is encountered and acknowledged.

\section{ELOG ALGORITHMS}

When the reset switch is released on the ELOG controller, program control is transferred to an initialization routine that presets the RAM and the microprocessor flags and then transfers control to either the data recording algorithm or the data transmission algorithm, depending on the state of the mode switch.

\section{Data Recording Algorithm}

The data recording function utilizes a background program, a tape-writing routine, a stop routine, and an interrupt service routine. Control initially is transferred to the background program, which simply monitors the amount of information in the data buffer and searches for an end-of-data signal that occurs when the 
timing switch is turned off. Control remains in the background program except when interrupts are serviced or a data buffer is transferred to cassette tape.

When the precision R.C timer "ticks" and an internpt is requested, program control is transferred to the interrupt service routine. This routine first reads the keys and checks for changes from the last reading. When a change appears, the previous keyboard configuration and the period of time that it lasted is entered in the data buffer and the duration "clock" maintained by the interrupt routine is reset to one. When no key change appears, the duration clock is incremented by one count. The clock is an 8-bit word, and when it overflows the overflow is entered in the data buffer and the clock is reset to one. After performing these functions, the interrupt routine transfers control back to the program location where the interrupt was requested.

When a full data buffer is encountered in the background program, control is transferred to the tapewriting routine. This routine first turns on the cassette recorder and waits $1.5 \mathrm{sec}$ for the recorder to warm up. It then transfers information word by word from the data buffer to the cassette tape. Information is transferred 4 bits at a time by getting the data word, rotating it to interchange the high 4 bits and the low 4 bits, transmitting the low bits, rotating the word, and transmitting the low bits again, and then incrementing the buffer pointer to the next word to be transmitted. A short delay is introduced between each 4-bit transmission to enable a cassette-reading routine to recognize boundaries between 4bit "words." When all the information is transferred from the data buffer, a buffer stop code is transmitted to cassette, the recorder is turned off, and program control is retumed to the background program.

When the timing switch is turned off, it is recognized in the background program, and control is transferred to the stop routine. This routine enters the final clock duration in the data buffer and transfers control to the tape-writing routine. The tape-writing routine is designed to recognize the stop mode and to respond by writing end-of-data transmission information in the buffer stop words and then retaining program control in an absorbing state.

\section{Data Transmission Algorithm}

The ELOG controller uses a single sequential routine in which the time from the last interrupt pulse determines whether the cassette tape is positioned within a word, between words, or at the end of a buffer. When the time between interrupts is shortest, the routine simply counts the pulse and keeps a record of the number of pulses in a row with the shortest interpulse interval. When a longer interval appears between pulses, the algorithm reflects the fact that a word boundary must have been reached by transforming the pulse count into a 4-bit data word, rotating it into the high 4 bits, and storing it in the data buffer. Two adjacent 4-bit words are packed together to form one 8-bit word before moving to the next buffer location. When the time between pulses exceeds the maximum interword interval, a complete buffer is assumed to have been read, and the ELOG controller turns off the cassette recorder and processes the data buffer.

The central computer uses a program to receive and verify data from the ELOG system and to transfer information back to the ELOG controller to allow proper processing of input data. Since this program is interactive with the routine used by the controller, description of the transmission algorithm includes both the controller function and the central computer function. After stopping the cassette recorder, two checks are performed on the data to determine its validity. If a data buffer contains fewer than five words, the controller considers it invalid and excludes it from the data run. In addition, the first two buffer words should contain the start code only in the first buffer of data processed. If no start code is found in the first buffer of data, the ELOG controller does not transmit the data and resumes searching the cassette for a buffer of data with the proper start code. If a verified start code is found in a subsequent buffer of data by the central computer, the data that has already been verified is aborted and a new data file is started. This check gives the ELOG system the capability of skipping past parts of records that may have been left on cassette tape from earlier data runs.

Data sent to the central computer resides in Bits $1-4$ of a 10-bit transmission "word" that includes start and stop bits. Bit 7 is set high to maintain compatibility with 64-character ASCII, and Bit 8 is set to odd parity. The complete data buffer is transmitted 4 bits at a time, and the ELOG controller waits for a verification character from the central computer, indicating receipt of the data. To be accepted and filed by the central computer, a complete buffer must be transmitted twice in a row in identical form, and the central computer continues to request retransmission of the data until two identical buffer transmissions have been sent in sequence. When the transmission criterion is met, the central computer signals the controller to go on to the next buffer. The controller then turns the cassette recorder on and processes the next buffer as described above. Data transmission continues until the central computer encounters and verifies a stop code and signals the controller to end transmission.

The above description of the ELOG data input system was prepared with the hope that future microprocessor-based designs might find this approach of some value in early planning. It also is hoped that the ELOG system can be made available to the behavioral 
science community in kit form for the price of the printed circuit board and components. Further information is available from the author.

\section{REFERENCE NOTES}

1. National Semiconductor Corporation. ISP-8A/600 single-chip 8-bit n-channel microprocessor [SC/MP-II] Publication No. 426305290-001A, Santa Clara. California. 1976.
2. Fitzpatrick. L. J. System and method of recording (vent information for subsequent analysis. U.S. Patent No. $3.944,796,1976$.

\section{REFERENCES}

Fitzpatrick. L. J. Automated data collection for observed events. Behavior Research Methods \& Instrumentation. 1977. 9. 447-451.

(Received for publication April 10, 1978; accepted April 11, 1978.) 